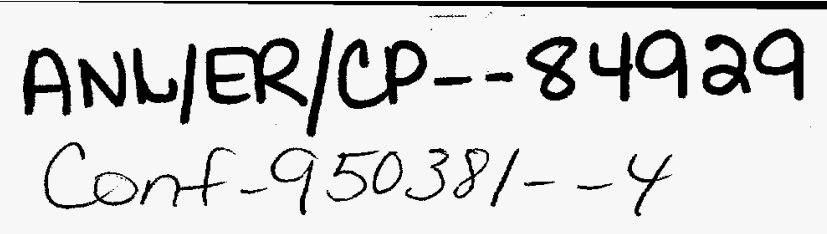

\title{
WAVELET ANALYSIS OF ATMOSPHERIC TURBULENCE STRUCTURES: WHAT CAN WE GAIN FROM THIS NEW TECHNIQUE?
}

\author{
Weigang Gao* \\ Environmental Research Division, Argonne National Laboratory, Argonne, Illinois \\ Bai-Lian Li \\ Department of Industrial Engineering, Texas A\&M University, College Station, Texas
}

\section{INTRODUCTION}

In recent years, the wavelet transform, a new mathematical tool, has found a wide range of applications in signal processing and data analysis (e.g., Farge, 1992). Similar to traditional spectral analysis with the Fourier transform, the wavelet transform unfolds a time series of data in a spectral domain by integrating the inner product of the data and a transfer function. However, unlike the Fourier transform, the wavelet transform allows the shape and size of the transfer function to change with frequency and can be used to calculate the energy contribution from different scales at a given location or time. Thus, the wavelet transform can provide a localized spectral analysis for an irregular data set and is especially valuable for identifying instantaneous characteristic structures hidden in the data.

The wavelet transform is defined by

$\mathrm{T}_{\mathrm{W}}(a, b, p)=\frac{1}{a^{p}} \int \mathrm{f}(\mathrm{t}) \psi\left(\frac{\mathrm{t}-b}{a}\right) \mathrm{dt}$

where the integration of the inner product of analyzed data $f(t)$ and the "mother wavelet" $\psi[(t-b) / a]$ is calculated for each point, indicated by $b$, of the data; $a$ is the dilation factor that gives the size of the mother wavelet and thus scale information; and $a p$, where typically $p$ $=0.5$ or 1.0 , is used to normalize transform coefficients. The distribution of the resulting transform coefficients over scale and location

Corresponding author address: Weigang Gao, Environmental Research Division, Bldg. 203, Argonne National Laboratory, Argonne, IL 60439 provides a two-dimensional picture of the relative strengths of different structures in the data.

The turbulent flow in the atmospheric boundary layer contains multiple scales, and under some conditions the turbulent transport of energy and mass can be dominated by certain organized motions with characteristic scales. Examples include thermal convective plumes with associated updrafts and surrounding downdrafts (Kaimal and Businger, 1970) and coherent structures with associated organized transport in the roughness sublayer near a plant canopy (Gao et al., 1989). Objectively identifying these structures from turbulence measurements is often difficult with the traditional statistical analysis and requires a special sampling technique (Gao et al., 1992). Analyses by Collineau and Brunet (1993), Gao and $\mathrm{Li}$ (1993), and Coulter and Li (1995) have shown that the wavelet transform is quite effective for detecting the coherent structures in turbulence data taken in the atmospheric boundary layer. The present paper demonstrates several specific methods of using the wavelet transform to study atmospheric turbulence.

\section{TIME-SCALE CROSS SECTION OF WAVELET TRANSFORM COEFFICIENTS}

Analysis of a data time series using (1) produces a set of transform coefficients changing with time $b$ and scale $a$. Figure $1 a$ shows the time-scale distribution of the wavelet coefficients calculated for the vertical velocity fluctuations (Fig. Ib) measured near the top of a deciduous forest (Gao et al., 1989). In this calculation, the mother wavelet, the second derivative of the Gaussian function (often 


\section{DISCLAIMER}

This report was prepared as an account of work sponsored by an agency of the United States Government. Neither the United States Government nor any agency thereof, nor any of their employees, makes any warranty, express or implied, or assumes any legal liability or responsibility for the accuracy, completeness, or usefulness of any information, apparatus, product, or process disclosed, or represents that its use would not infringe privately owned rights. Reference herein to any specific commercial product, process, or service by trade name, trademark, manufacturer, or otherwise does not necessarily constitute or imply its endorsement, recommendation, or favoring by the United States Government or any agency thereof. The views and opinions of authors expressed herein do not necessarily state or reflect those of the United States Government or any agency thereof. 


\section{DISCLAIMER}

Portions of this document may be illegible in electronic image products. Images are produced from the best available original document. 
referred to as the "Mexican hat") has the form $\psi\left(t^{\prime}\right)=\left(1-16 t^{2}\right) \exp \left(-8 t^{\prime 2}\right)$, where $t^{\prime}=(t-$ b) /a. In Fig. Ia, the horizontal axis represents the time of data sampling, and the vertical axis represents the scale at which the coefficients were calculated. Magnitudes of the coefficients indicate the relative strengths of structures at

(a)

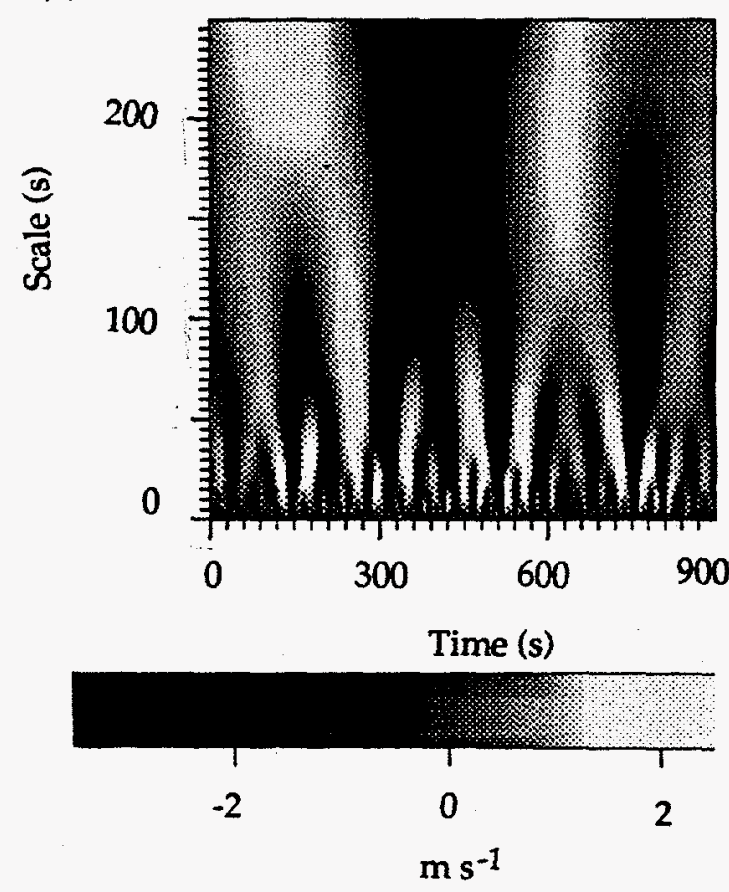

(b)

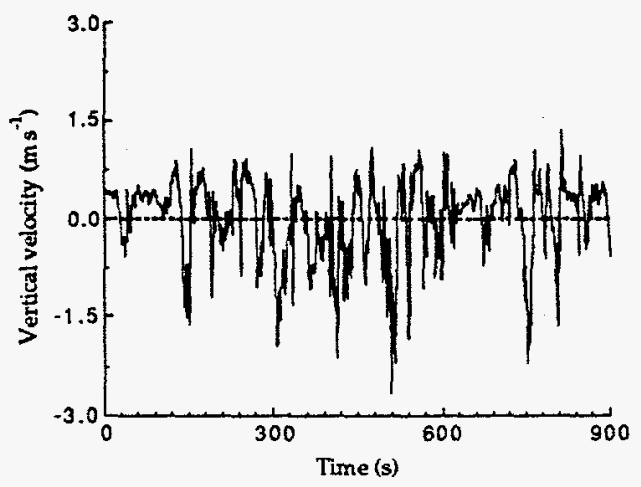

Figure 1 (a) Wavelet transform coefficients for a $15-$ min period of turbulent fluctuations in the vertical velocity measured at the top of a deciduous forest. (b) The corresponding time series of the same data. The scale in (1a), calculated as $0.5 a$, represents the duration of updraft or downdraft. different scales and times. For this specific example, discrete, plume-like patterns about 40$50 \mathrm{~s}$ in scale can easily be identified. These localized centers appear to provide a smooth transition between the smaller-scale motions indicated by the coefficients near the bottom of Fig. 1a and the larger-scale circulations (>200 s). Gao et al. (1989) described in detail the characteristics of these coherent structures in terms of vertical velocity and temperature fields. The coherent structures efficiently transport energy, momentum, and trace gases between the atmosphere and the forest canopy. The twodimensional display in Fig. 1a provides a good visualization and qualitative identification of these important structures hidden in the raw data.

\section{WAVELET VARIANCE}

To further examine the scale dependence of the turbulence structure and to objectively detect scales associated with the coherent structure, the total variance can be calculated by integrating the squares of the coefficients for all data points. Collineau and Brunet (1993) demonstrated that this integration is proportional to the variance of the data and the energy contained within each scale. Figure 2 shows the scale dependence of the wavelet variance for the data shown in Fig. 1. For the curve calculated with $p=0.5$, the variance has a peak near the scale of $45 \mathrm{~s}$, which agrees with the display in Fig. 1a. The scale represents the duration of updraft or downdraft, according to the definition of the transfer function (Gao and

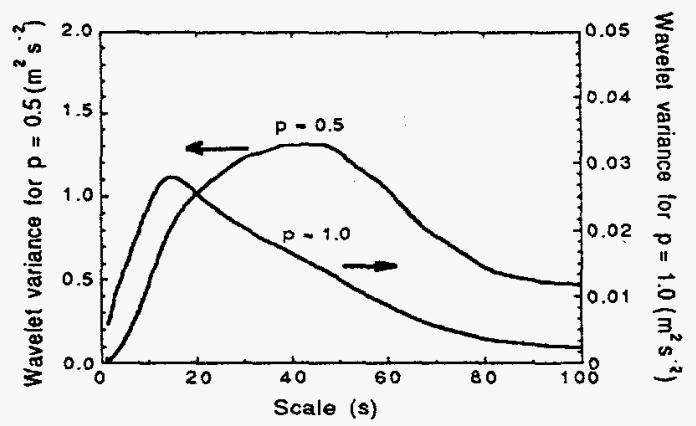

Figure 2. Wavelet variance of vertical velocity calculated for data in the 30 -min period including the 15-min period shown in Fig. 1, by using two different $p$ values. 
$\mathrm{Li}, 1993)$. However, another peak near the scale of $13 \mathrm{~s}$ that is not detected when $\mathrm{p}=0.5$ appears when $p=1.0$. A bimodal variance distribution also appears in the vertical velocity measured at other heights (Gao and Li, 1993). Calculation of the correlation between transformed vertical velocity and temperature indicates that only the peak at $45 \mathrm{~s}$ is associated with strong heat transfer, one of the characteristics of the coherent structure. Values of 0.5 and 1.0 were used for $p$ to ensure energy conservation in the wavelet analysis (Collineau and Brunet, 1993). The result here, however, suggests that the choice of $p=0.5$ in wavelet variance analysis emphasizes larger structures but misses structures with smaller scales. Thus, multiple analyses with different choices for $p$ might be necessary to capture all significant variance peaks and associated scales, and the following wavelet correlation might be useful in determining which peak is responsible for the coherent structure.

\section{CROSS CORRELATION OF TRANSFORM COEFFICIENTS}

Important characteristics of coherent structures in the atmospheric boundary layer can also be examined by using the cross correlation of transform coefficients. The wavelet correlation, which is different from the conventional correlation calculation, shows the relative contributions of phenomena at different scales. The wavelet correlation can be calculated for time series of two different variables measured at the same height or for time series of a single variable measured at different heights. The former is important for understanding relationships between flow and scalar fields associated with the coherent structure, and the latter is useful for determining the spatial coherence of the structure. Figure $3 a$ shows the cross correlation of transformed vertical velocity and temperature measured simultaneously at the same height near the canopy top. The horizontal axis is the time lag or phase difference used in the calculation, and the vertical axis is the scale. The cross correlation has a positive maximum near the scale of $45 \mathrm{~s}$ with a slight time lag and also a negative maximum at a similar scale but with a phase shift of about $50 \mathrm{~s}$. The positive maximum is probably associated with warm updraft and cool (a)

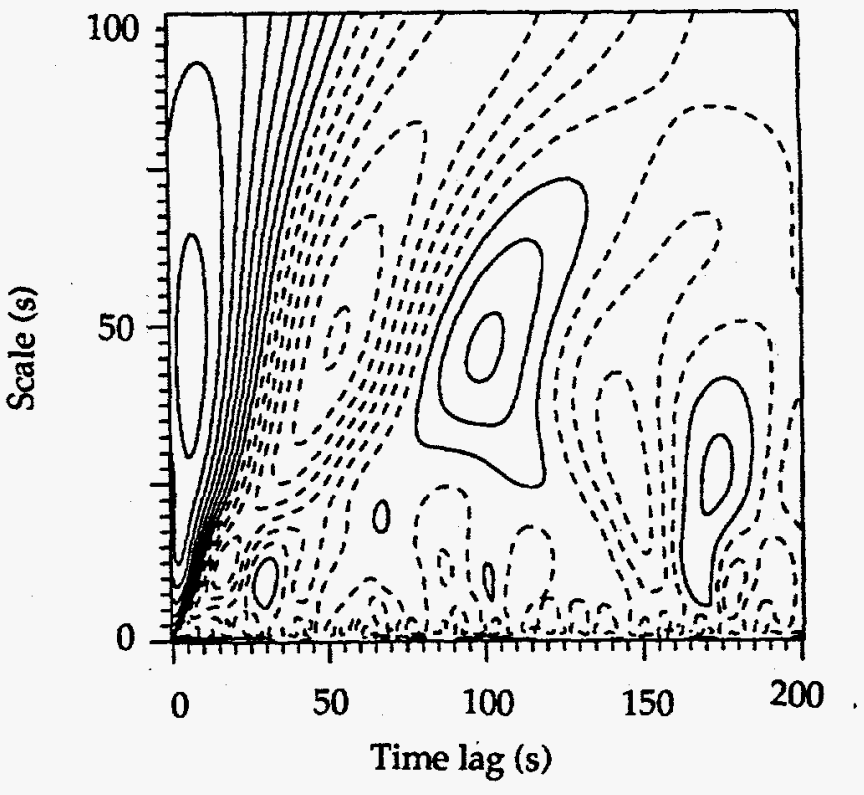

(b)

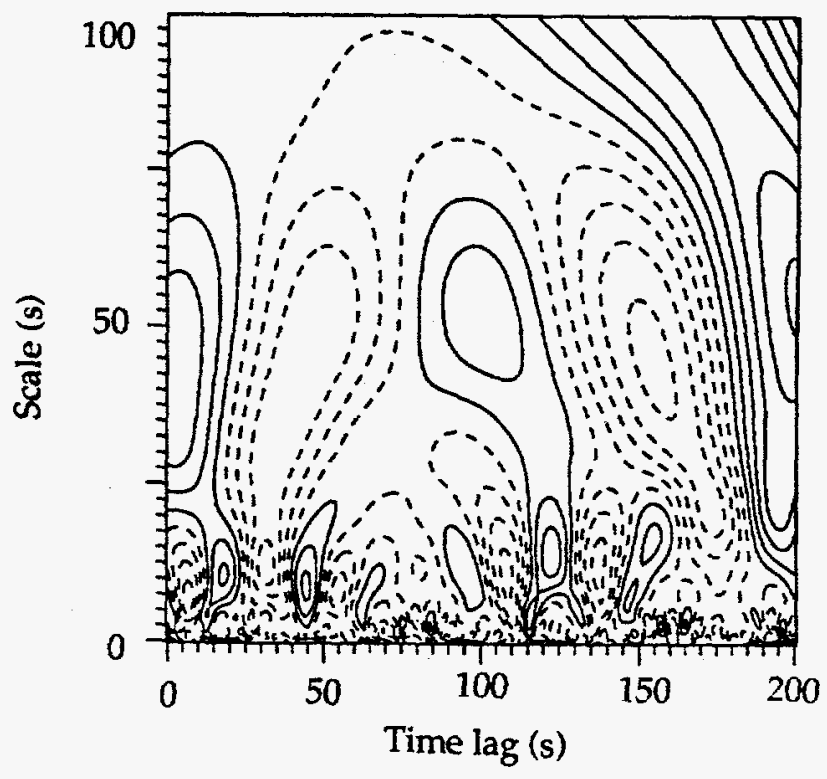

Figure 3. Cross correlation of wavelet transform coefficients (a) between vertical velocity and temperature at the same height (canopy top) and (b) between the vertical velocities measured at the canopy top and 2.5 times the canopy height. Positive and negative values are denoted by solid and dashed lines, respectively. The contour intervals are 0.1 in (3a) and 0.05 in (3b), respectively. 
downdraft regions, while the negative correlation maximum may be contributed by the correlation of adjacent updrafts and downdrafts. Thus, the time shift of $50 \mathrm{~s}$ may indicate the horizontal transport time between the core of a warm updraft and the core of the compensating downdraft. The correlation at the smaller scale near $13 \mathrm{~s}$, corresponding to the peak in Fig. 2, is not as strong, implying a less efficient heat transfer.

Figure $3 \mathrm{~b}$ shows the correlation of transform coefficients for the time series of vertical velocity measured at the canopy height and about 2.5 times the canopy height. This spatial correlation also has positive and negative maxima near the scale of $45 \mathrm{~s}$, indicating the strong spatial coherence at this scale. The several local centers appear near the scale of 13 $s$, which corresponds to the peak variance shown in Fig. 2. What causes the vertical coherence at this smaller scale is not fully understood at this time.

\section{CONCLUSIONS}

We examined three specific methods for using the wavelet transform to derive information on the turbulence structure in the atmospheric boundary layer from raw data. The scale-time cross section method yields a qualitative picture of turbulence structures occurring at different times and different scales, the wavelet variance method provides a relatively objective estimate of principal scales associated with organized structures, and the wavelet cross correlation helps in identifying motions that are most important in turbulent transport and spatial coherence. The wavelet analysis can also be used to decompose the data for different scales and to detect coherent structures objectively by using the transform coefficient at the principal scale detected by the variance method (Collineau and Brunet, 1993; Coulter and $\mathrm{Li}, 1995)$. These methods can improve our capability to unfold the data for hidden information that is difficult to obtain from traditional spectral analysis and thus can provide a useful tool for understanding multiscale phenomena in the atmospheric boundary layer.

\section{ACKNOWLEDGMENTS}

This work was supported by the U.S. Department of Energy, Office of Energy Research, Office of Health and Environmental Research, under contract W-31-109-Eng-38 and by the Laboratory-Directed Research and Development Program.

\section{REFERENCES}

Collineau, S., and Y. Brunet, 1993: Detection of turbulent motion in a forest canopy. Part I: Wavelet analysis. Boundary-Layer Meteorol., 65, 357-379.

Coulter, R. L., and B. L. Li, 1995: A technique using the wavelet transform to identify and isolate coherent structures in the planetary boundary layer (these proceedings).

Farge, M., 1992: Wavelet transforms and their applications to turbulence. Annu. Rev. Fluid Mech., 24, 395-457.

Gao, W., R. H. Shaw, and K. T. Paw U, 1989: Observation of organized structures in turbulent flow within and above a forest canopy. Boundary-Layer Meteorol., 47, 349-377.

Gao, W., R. H. Shaw, and K. T. Paw U, 1992: Conditional analysis of temperature and humidity microfronts and ejection/sweep motions within and above a deciduous forest. Boundary-Layer Meteorol., 59, 3557.

Gao, W., and B. L. Li, 1993: Wavelet analysis of coherent structures at the atmosphereforest interface. J. Appl. Meteorol., 32, 1717-1725.

Kaimal, J. C., and J. A. Businger, 1970: Case studies of a convective plume and dust devil. J. Appl. Meteorol., 9, 612-620. 